\title{
A biomechanical comparison of a mesh suture to a polyblend suture in a porcine tendon model
}

\author{
Zhanwen Wang ${ }^{1,2}$, Zeling Long ${ }^{1}$, Hong Li ${ }^{1}$, Hongbin Lu ${ }^{2}$, Anne Gingery ${ }^{1}$, Peter C. Amadio ${ }^{1}$, \\ Steven L. Moran ${ }^{1}$, Chunfeng Zhao ${ }^{1}$ \\ ${ }^{1}$ Department of Orthopedic Surgery, Mayo Clinic, Rochester, MN, USA; ${ }^{2}$ Department of Sports Medicine, Xiangya Hospital, Central South \\ University, Changsha, China \\ Contributions: (I) Conception and design: Z Wang, C Zhao; (II) Administrative support: C Zhao; (III) Provision of study materials or patients: Z \\ Long, H Li; (IV) Collection and assembly of data: Z Wang, Z Long; (V) Data analysis and interpretation: H Lu, A Gingery, PC Amadio, SL Moran; \\ (VI) Manuscript writing: All authors; (VII) Final approval of manuscript: All authors. \\ Correspondence to: Chunfeng Zhao, MD. Department of Orthopedic Surgery, Mayo Clinic, Rochester, MN 55905, USA. \\ Email: Zhao.Chunfeng@mayo.edu.
}

Background: The suture-tendon interface turned out to be the weak point of a repaired rotator cuff. A double rip-stop (DRS) technique was developed to enhance the strength of the suture-tendon interface. The first aim of this study was to compare the suture-tendon interface strength between mesh suture and the No. 2 FiberWire (FW), which is commonly used in the clinic. The second aim was to compare the biomechanical properties of rotator cuff repair between mesh suture and No. 2 FiberWire using a typical suture-bridge (SB) and DRS techniques.

Methods: Eighteen porcine subscapularis tendon (SST) was randomly assigned to the Mesh-tendon group and FiberWire-tendon group. A single suture loop was passed through the SST with a Mesh suture or FiberWire. Thirty-two infraspinatus tendons (ISTs) were randomly assigned to four groups: SB-Mesh group: SB technique with Mesh suture, SB-FW group: SB technique with FiberWire, DRS-Mesh group: DRS technique with Mesh suture, and DRS-FW group: DRS technique with FiberWire. All repaired specimens were underwent failure testing. Failure modes, load to create a 3-mm gap, failure load, and stiffness were compared.

Results: There were no significant differences between the Mesh-tendon group and FiberWire-tendon group regarding the failure load, stiffness, and ultimate stress. When the same technique was used, the rotator cuff repaired with a mesh suture had the similar load to create a 3-mm gap, failure load, and stiffness compared with FiberWire. When the same suture was used, the DRS technique had a significantly higher load to create a 3-mm gap formation and failure load compared with the SB technique.

Conclusions: The repair failure strength and stiffness using the mesh suture were similar to the FiberWire suture regardless of the repair techniques. However, the repair strength in the DRS technique was significantly stronger than the SB technique when the same suture material was used.

Keywords: Rotator cuff; mesh suture; suture bridge technique; rip-stop; suture-tendon interface

Submitted Jan 28, 2020. Accepted for publication Aug 27, 2020.

doi: 10.21037/atm-20-1065

View this article at: http://dx.doi.org/10.21037/atm-20-1065 


\section{Introduction}

The incidence of rotator cuff tears increases along with age (1). Surgical repairs are often needed to reattach the torn tendon to its bony insertion. With the development of surgical techniques, most rotator cuff tears are repaired with the arthroscopic approach. However, the tendon healing capacity decreases with age increased $(2,3)$. Boileau et al. reported that the healing rate was only $43 \%$ for patients older than 65 years after single-row repair (2). Meanwhile, the poor quality of rotator cuff tendon is also related to the high retear rate after repair (4). Whereas many biologic augmentations such as scaffolds, bioactive factors, and stem cells have been widely investigated, the results are controversial (5-11). This indicated that the high retear rate could not be fully ascribed to poor biological healing after rotator cuff repair. Due to delayed healing in the elderly, the primary fixation strength after surgery becomes crucial to maintain the repair integrity for the healing process. Anchors, suture materials, as well as suture configuration have been shown to play an important role in the primary fixation strength (12-14).

Recently, a mesh suture proposed for abdominal wall repair showed promising results with improved early strength (15-18). Afterwards, mesh suture has been investigated in the human cadaveric flexor tendons and the canine cadaveric rotator cuff repair $(19,20)$. Zhang et al. showed that rotator cuff repaired with a mesh suture significantly increased the failure load compared to the traditional polydioxanone suture II or FiberWire sutures (19). However, the study by Zhang et al. used transosseous technique rather than the suture anchor system, which is commonly used for arthroscopic surgery. Whether suture anchors could be loaded with mesh suture and used for rotator cuff repair have not been studied. The suture bridge (SB) technique has been widely used for rotator cuff repair in arthroscopic surgery. However, many biomechanical studies showed that the repaired tendon was cut through by the suture during the failure test $(21,22)$. In order to enhance the suture-tendon interface, "rip-stop" configuration was developed and showed stronger biomechanical performance (23-25). Previously, the rip-stop structure was created on the bursal side of the tendon during a rotator cuff surgery procedure. Here we modified the current "rip-stop" technique and developed a double rip-stop (DRS) technique to further enhance the strength of suture-tendon interface. Therefore, the first aim of this study was to compare the suture-tendon interface strength between mesh suture and the No. 2 FiberWire, which is commonly used in the clinic. The second aim was to compare the biomechanical properties of the rotator cuff repair between the mesh suture and No. 2 FiberWire using two repair techniques (SB and DRS). The hypothesis is that the mesh suture has better biomechanical performance on the suture-tendon interface compared to the No. 2 FiberWire, and the rotator cuff repaired with Mesh suture had better biomechanical properties than the No. 2 FiberWire, regardless of repair technique. We present the following article in accordance with the MDAR reporting checklist (available at http://dx.doi.org/10.21037/atm-20-1065).

\section{Methods}

\section{Study design}

Thirty-two shoulders were harvested from mature pigs (average weight, $60 \mathrm{~kg}$ ) and sacrificed for other studies, which was approved by the Institutional Animal Care and Use Committee. This study was carried out in two parts. Part 1: eighteen subscapularis tendons (SSTs) were randomly assigned to a 0 sized mesh suture or a No. 2 FiberWire suture. A single suture loop was placed through the SST, followed by biomechanical testing (Figure 1). Part 2: thirty-two infraspinatus tendons (ISTs) were used for rotator cuff repair and randomly assigned to four groups. Then the repaired rotator cuffs were biomechanically tested (Figure 1).

\section{Sutures}

Duramesh Suturable Mesh was provided by Mesh Suture Inc., Dorado, PR. Mesh suture has a cylindrical hollow and cylindrical shape and is fabricated by braiding twelve slender polypropylene filaments which are approximately 125 microns in diameter. Each filament is bonded to the others at every contact point (20) with the final outer diameter of unstressed suture measuring $2.59 \mathrm{~mm}$. The open structure of the mesh suture allows the filaments to collapse at the knot to limit its size. No. 2 FiberWire (Arthrex, Naples, FL) consists of a core of several small individual strands of polyethylene covered with braided polyester suture material. The diameter of No. 2 FiberWire is $0.69 \mathrm{~mm}$ (26). After a rotator cuff repair, the diameter of 0 sized mesh suture decreased to about $1 \mathrm{~mm}$.

\section{Part 1}

\section{Suture-tendon specimen preparation}

After the SST was identified and carefully isolated, the SST 

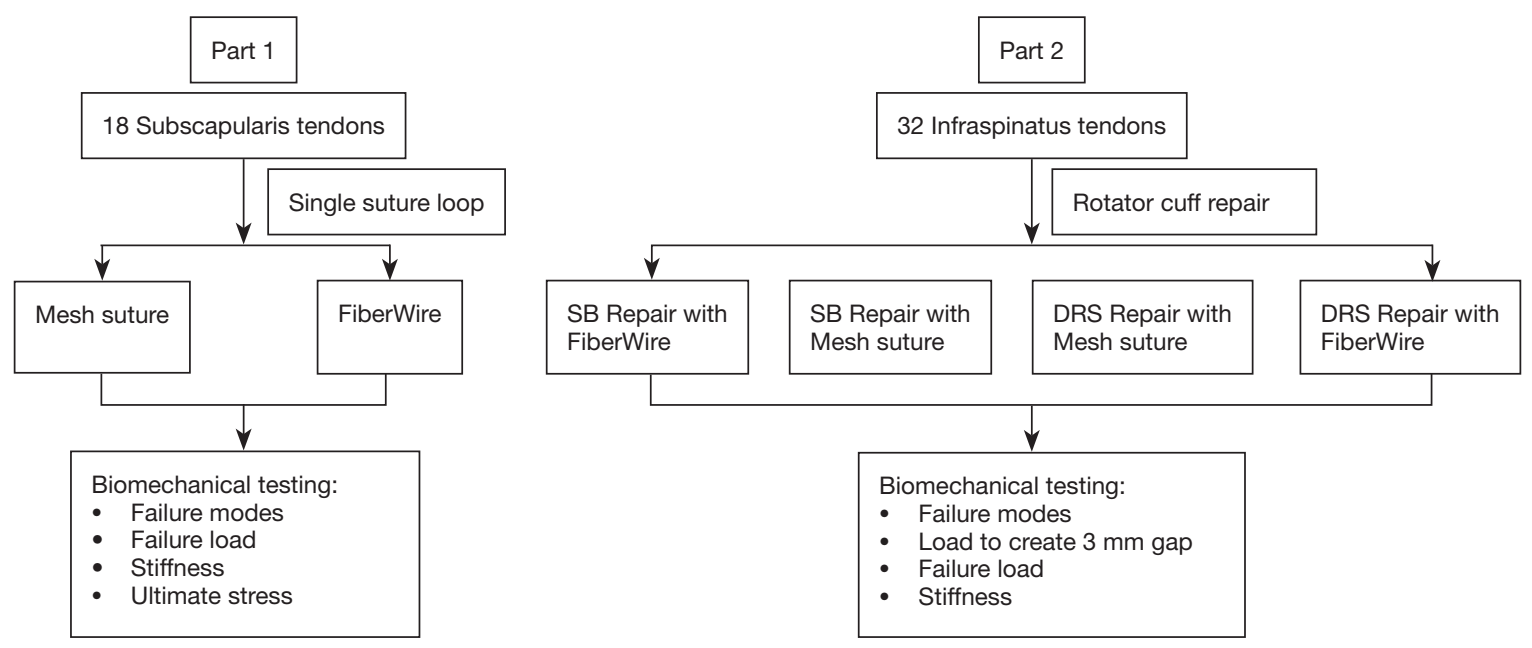

Figure 1 Flow chart of the study design. SB, suture bridge technique; DRS, double rip-stop technique.
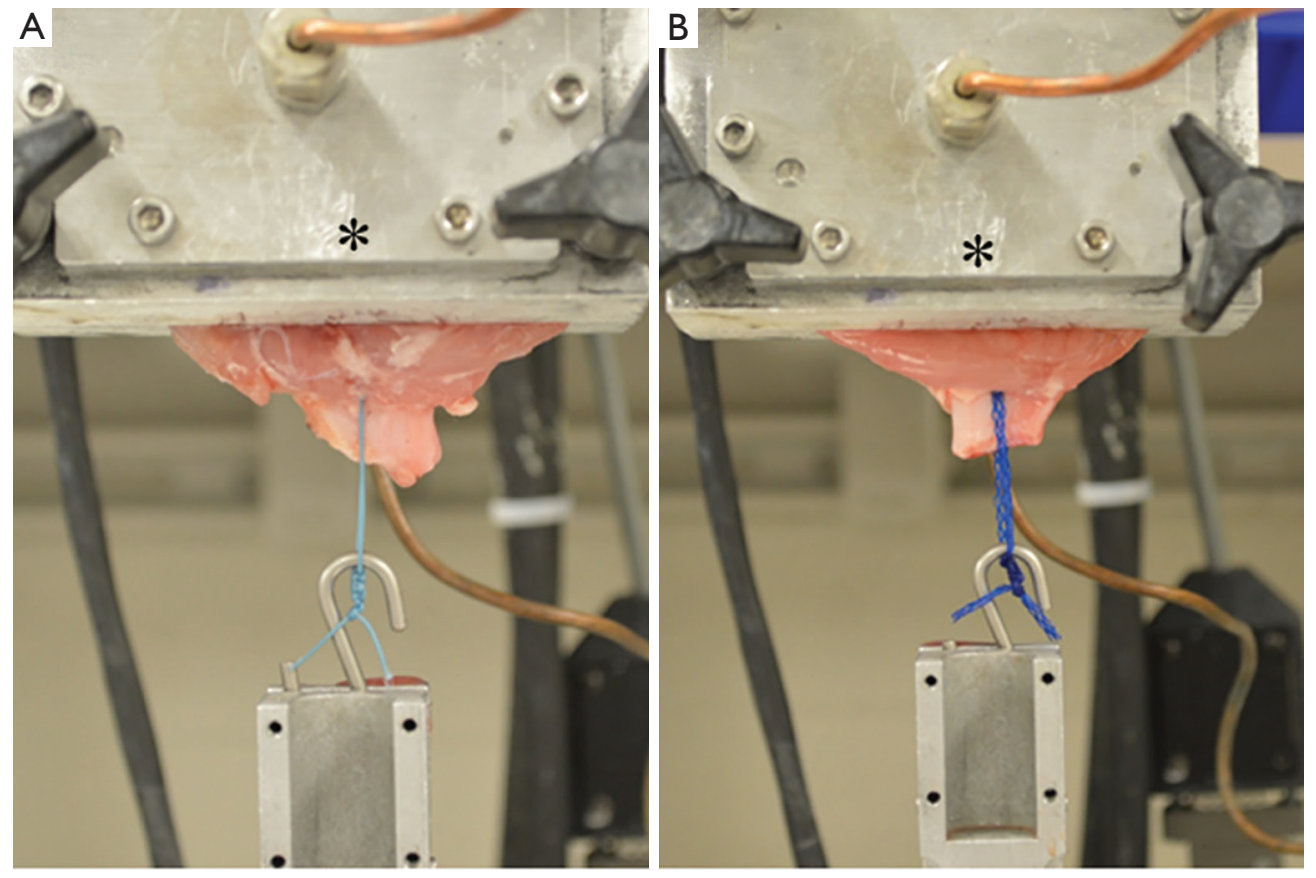

Figure 2 Biomechanical testing setup for suture-tendon specimens of No. 2 FiberWire group (A) and Mesh suture group (B). * denotes the custom-made clamp.

was sharply transected at the bony insertion site. The thin part of SST on the insertion was trimmed. Either Mesh suture (size 0) or No. 2 FiberWire was passed in a simple fashion through the SST at $1 \mathrm{~cm}$ from the tendon edge (Figure 2). Five square knots were tied to form a $3-\mathrm{cm}$ suture loop $(12,13)$. The thickness of the SST at the entry where the suture passed through and the length between the suture entry and the SST edge were measured with a digital caliper (Johnson Level \& Tool Mfg. Co., Inc. WI, USA).

\section{Biomechanical testing}

The suture-tendon specimens were loaded to the MTS system (MTS-312, Eden Prairie, Minnesota). The subscapularis muscle was gripped in a custom-made 

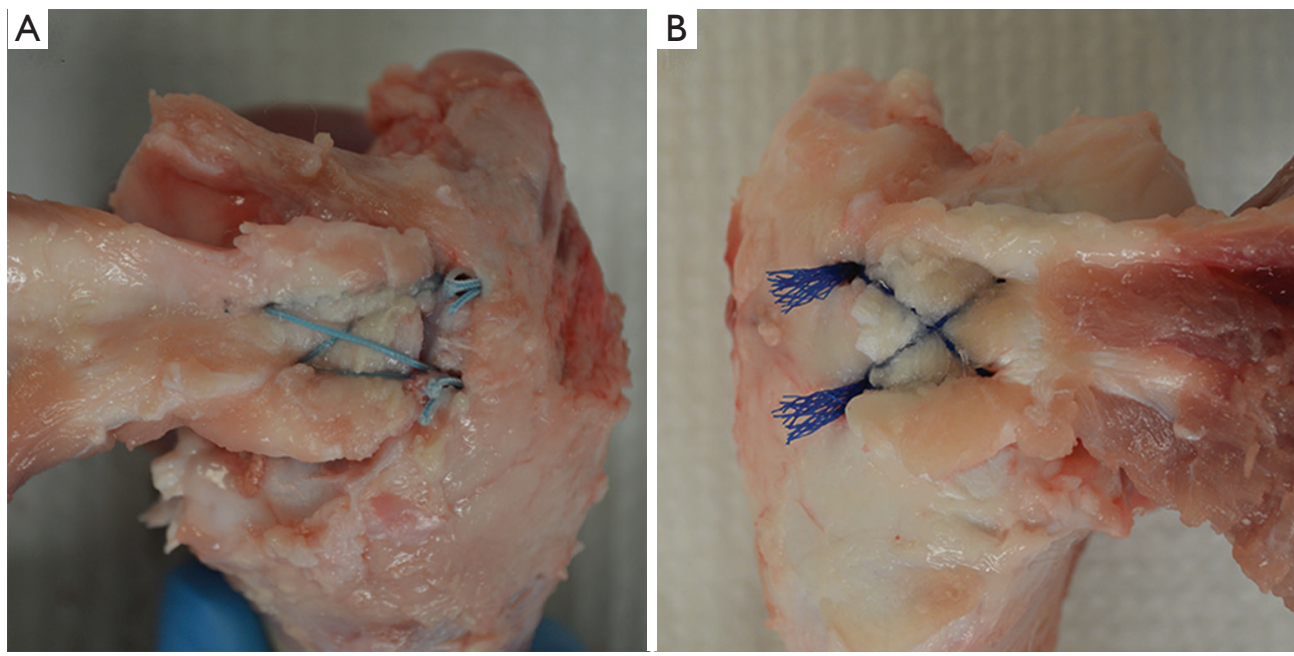

Figure 3 Final configuration of the suture bridge technique using a No. 2 FiberWire (A) and mesh suture (B).

clamp and frozen by liquid CO2 (Figure 2). All samples were loaded to failure at a rate of $3 \mathrm{~mm} / \mathrm{s}(27)$. Loaddisplacement was recorded and failure load was defined as the peak force recorded. Stiffness was calculated from the slope of the linear region of the load-displacement curve. The ultimate stress was calculated by dividing the failure load with the area of tendon spanned by suture. The failure modes were recorded for each specimen. The tendon tissues were kept moist using isotonic saline $(0.9 \%$ sodium chloride).

\section{Part 2}

\section{Rotator cuff repair}

The IST was then sharply dissected with a scalpel from its bony insertion on the greater tuberosity to simulate a fullthickness rotator cuff tear (28-31). The tendon thickness and the footprint dimensions at the distal and proximal end of the bony insertion were obtained with a digital caliper. The IST tendons were randomly assigned to four groups: (I) suture bridge technique with mesh suture (SB-Mesh) group; (II) suture bridge technique with No. 2 FiberWire (SB-FW) group; (III) DRS technique with Mesh suture (DRS-Mesh) group; and (IV) DRS technique with No. 2 FiberWire (DRS-FW) group. Two 4.5-mm medial suture anchors (BioComposite Corkscrew FT Anchor, loaded with No. 2 FiberWire, Arthrex, Naples, FL) and two $3.5-\mathrm{mm}$ lateral anchors (BioComposite Pushlock, Arthrex, Naples, FL) were used for each repair. The tendon tissues were kept moist using isotonic saline ( $0.9 \%$ sodium chloride).

\section{Suture bridge technique with No. 2 FiberWire or mesh suture}

The SB repair was performed per the manufacturer's instructions. For the SB-FW group, two $4.5-\mathrm{mm}$ medial anchors were inserted laterally to the articular margin, $10 \mathrm{~mm}$ apart in the superior-inferior dimension, in a $45^{\circ}$ deadman's angle (32). The IST was reduced and perforated 12-14 mm medially by two suture limbs of each anchor. One suture limb from each of the medial row anchors was secured to the greater tuberosity with two knotless PushLock anchors. The lateral row anchors were inserted $20 \mathrm{~mm}$ lateral from the medial anchors (Figure $3 A$ ). The sutures were pre-tensioned with the slits of the driver for the Pushlock anchor before lateral row fixation.

For the SB-Mesh group, the No. 2 FiberWire in the suture anchor was replaced with a mesh suture, then the IST was repaired in the same way as the SB-FW group (Figure 3B).

\section{DRS technique with No. 2 FiberWire or mesh suture group}

The medial row anchors were placed as described in SB repair (Figure $4 A$ ). After two suture limbs from one anchor passing through the tendon (Figure 4B), one limb passed the tendon from the bursal surface to the articular surface (Figure $4 C$ ). A loop was formed on the surface of the tendon (Figure 4C). After passing laterally from the anchor (Figure $4 D$ ), this limb passed through the tendon from the articular surface to the bursal surface (Figure 4E). A ripstop was formed at the articular surface. Finally, this limb passed through the loop to develop the second rip-stop 

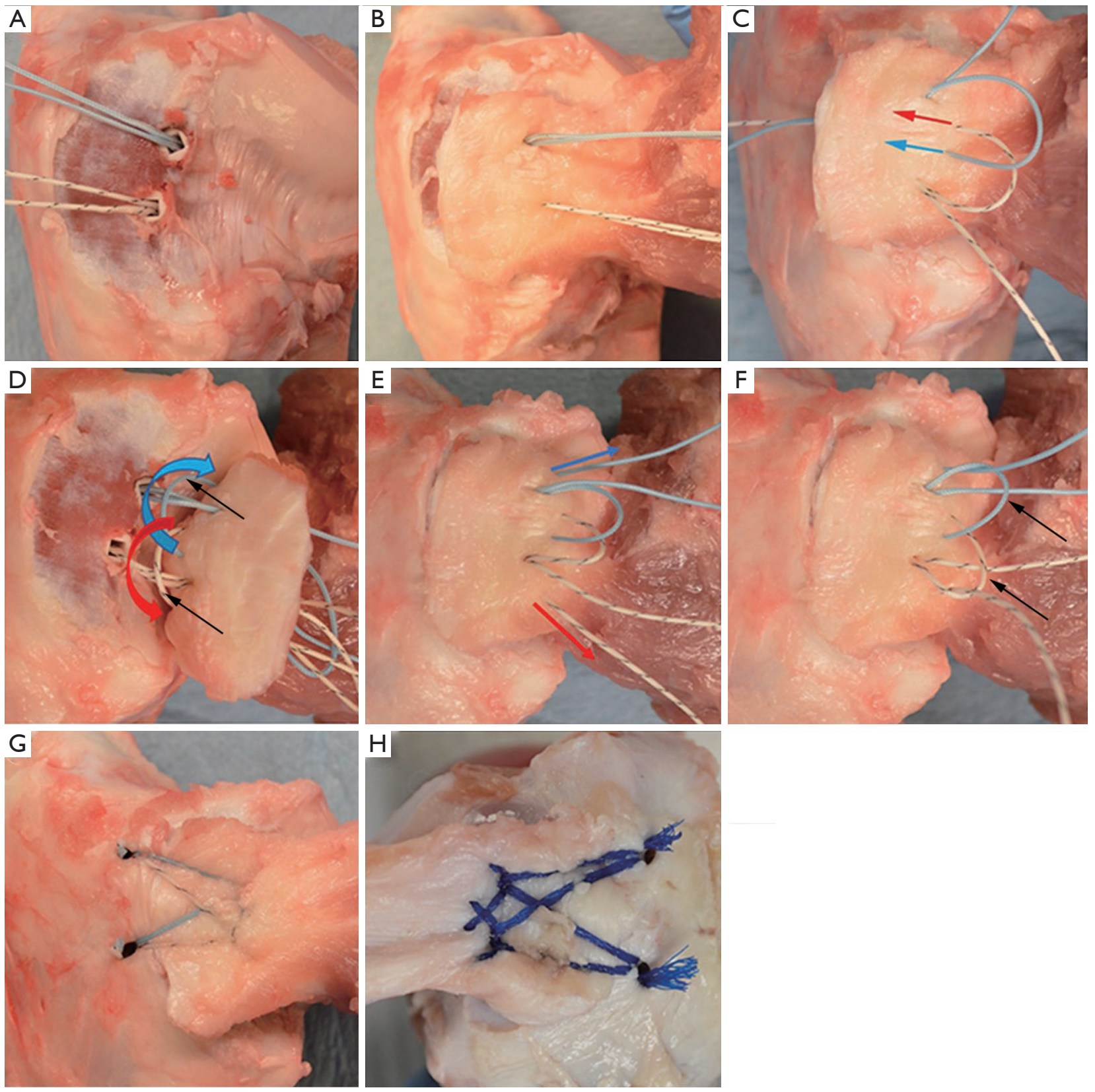

Figure 4 Surgical procedure and final configuration of the double rip-stop technique. Two medical anchors were inserted at the articular margin (A). Suture limbs of each anchor passed through the tendon (B). Then one limb passed the tendon from bursal surface to articular surface $(\mathrm{C})$ to form a loop on the surface of the tendon $(\mathrm{C})$. After passing laterally from the anchor (D), this limb passed through the tendon from articular surface to bursal surface (E). A rip-stop was formed at the articular surface (D). Finally, this limb passed through the loop to develop the second rip-stop (F). The sutures from the other anchor perforated the tendon in the same way. Thus, two rip-stops were formed on at the articular side (D), as well as the bursal side (F). The establishment of the lateral row was the same as the SB repair. The final configuration of double rip-stop technique using a No. 2 FiberWire $(\mathrm{G})$ and a mesh suture (H). Black arrows denote the rip-stop. Blue and red arrows denote the direction of suture passing through tendon for the blue and white suture limb respectively. 
Table 1 Measurement of suture-tendon specimens

\begin{tabular}{lccc}
\hline Variable & Mesh suture & FiberWire & P value \\
\hline Tendon thickness $(\mathrm{mm})$ & $1.88 \pm 0.27$ & $1.96 \pm 0.22$ & 0.56 \\
$\begin{array}{l}\text { Distance between suture } \\
\text { entry and tendon edge }(\mathrm{mm})\end{array}$ & $10.90 \pm 0.95$ & $10.75 \pm 0.34$ & 0.67 \\
$\begin{array}{l}\text { Area of tendon spanned by } \\
\text { suture }\left(\mathrm{mm}^{2}\right)\end{array}$ & $20.39 \pm 2.73$ & $21.03 \pm 2.67$ & 0.65 \\
\hline
\end{tabular}

Data were shown as mean \pm standard deviation.

(Figure 4F). The sutures from the other anchor perforated the tendon in the same way. Thus, two rip-stops were formed on at the articular side (Figure $4 D$ ), as well as the bursal side (Figure $4 F$ ). The establishment of the lateral row was the same as the SB repair (Figure $4 G, H)$.

\section{Biomechanical testing}

After the rotator cuff repair, the humerus was mounted into a plastic tube with bone cement and positioned to a vertical load application in the MTS system (MTS312, Eden Prairie, Minnesota). The infraspinatus muscle was gripped in the same way as the subscapularis muscle. Before biomechanical testing, the specimen was marked at (I) medially to the medial row, (II) in between both suture rows, and (III) to the greater tuberosity. The repaired rotator cuff specimens were loaded to failure at a rate of $50 \mathrm{~mm} / \mathrm{min}$. Load-displacement was recorded and failure load was defined, as the peak force recorded. Stiffness was calculated from the slope of the linear region of the loaddisplacement curve. The failure modes were recorded for each specimen. During the failure testing, videos were recorded for each specimen and processed with Image J.

\section{Statistical analysis}

Continuous data were presented as the mean \pm standard deviation (19). The Student $t$-test was used to compare the SST thickness, distance between the suture entry, and SST tendon edge, failure load, stiffness, and ultimate stress between the mesh suture-tendon group and FiberWiretendon group. The two-way ANOVA with Bonferroni test was used to compare the footprint and tendon dimension of IST, failure load, stiffness, and load to form a 3-mm gap among the rotator cuff repaired groups. Fisher's exact test was used to compare the incidence rate of failure modes. The level of significance was set at $\mathrm{P}<0.05$.

\section{Results}

\section{Suture-tendon specimens}

\section{Specimen consistency}

There was no significant difference between the mesh suture and the No. 2 FiberWire groups, with respect to the tendon thickness at the site of the suture passing through, the distance between the suture entry site and tendon edge, and the cross-section area of tendon (Table 1).

\section{Biomechanical properties}

All suture-tendon specimens were failed by the suture cutting through the SST. The failure load of the Mesh suture group was $54.1 \pm 22.5$ and $53.6 \pm 15.2 \mathrm{~N}$ for FiberWire group. The stiffness of the Mesh suture group was $2.4 \pm 0.5$ and $2.6 \pm 1.2 \mathrm{~N} / \mathrm{mm}$ for FiberWire group. The ultimate stress of the mesh suture group was $2.4 \pm 0.9$ and $2.6 \pm$ 0.7 MPa for FiberWire group. There was no significant difference between the mesh suture and the FiberWire groups regarding the failure load, stiffness, and ultimate stress (Figure 5).

\section{Repaired rotator cuff specimens}

\section{Footprint dimensions and tendon thickness}

There were no grossly abnormal anatomies found in all the samples. There was no significant difference among the four groups $(\mathrm{P}>0.05)$ regarding the tendon thickness, footprint dimensions, or insertional areas (Table 2).

\section{Biomechanical properties}

\section{Load to create $3 \mathrm{~mm}$ of gap formation}

The mean load to create a $3-\mathrm{mm}$ gap between the markers on the medial tendon and the humerus was $57.0 \pm 19.6 \mathrm{~N}$ for the SB-Mesh group, $80.8 \pm 20.1 \mathrm{~N}$ for SB-FW group, $108.3 \pm 53.9 \mathrm{~N}$ for the DRS-Mesh group, and $126.9 \pm 32.5 \mathrm{~N}$ for the DRS-FW group (Figure 6A). There was no significant difference between the mesh and the FiberWire groups when the same suture technique was used. However, the DRS technique significantly improved the repair strength compared to the SB technique regardless of the suture materials $(\mathrm{P}<0.05$ for all).

\section{Modes of failure}

The failure modes of the repaired rotator cuff were summarized in Table 3. All samples in the SB-FW and SB- 

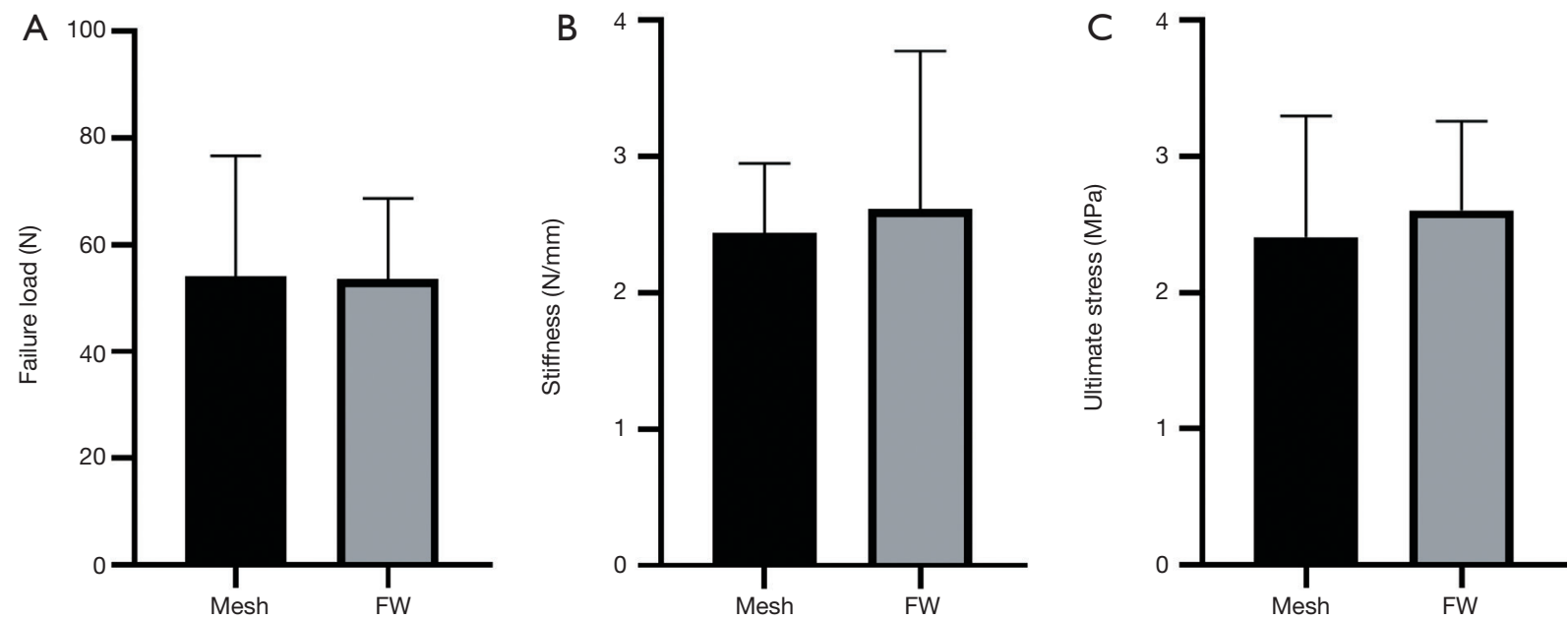

Figure 5 Comparison of the failure load (A), stiffness (B), and ultimate stress (C) of mesh suture-tendon group and the No. 2 FiberWiretendon group. Data were shown as mean \pm standard deviation. FW, FiberWire.

Table 2 Footprint and tendon dimensions for each technique

\begin{tabular}{lccccc}
\hline Technique & Tendon caliber medially $(\mathrm{mm})$ & $\begin{array}{c}\text { Tendon caliber } \\
\text { laterally }(\mathrm{mm})\end{array}$ & $\begin{array}{c}\text { Footprint } \\
\text { width }(\mathrm{mm})\end{array}$ & $\begin{array}{c}\text { Footprint } \\
\text { length }(\mathrm{mm})\end{array}$ & $\begin{array}{c}\text { Insertional } \\
\text { area }(\mathrm{mm})^{2}\end{array}$ \\
\hline SB-Mesh & $3.61 \pm 0.22$ & $1.35 \pm 0.15$ & $13.82 \pm 0.92$ & $20.72 \pm 1.58$ & $287.02 \pm 36.78$ \\
SB-FW & $3.37 \pm 0.38$ & $1.23 \pm 0.12$ & $14.34 \pm 1.13$ & $19.69 \pm 0.79$ & $282.45 \pm 26.63$ \\
DSR-Mesh & $3.40 \pm 0.23$ & $1.16 \pm 0.22$ & $14.22 \pm 0.93$ & $20.03 \pm 0.96$ & $285.21 \pm 26.66$ \\
DSR-FW & $3.55 \pm 0.52$ & $1.37 \pm 0.22$ & $14.50 \pm 0.65$ & $19.96 \pm 0.86$ & $289.52 \pm 20.75$ \\
\hline
\end{tabular}

Data were shown as mean \pm standard deviation. SB, suture-bridge; DRS, double rip-stop; FW, FiberWire.

Mesh groups were failed due to the suture cutting through the tendon, except one specimen in the SB-Mesh group, which failed by suture breakage. Four samples in the DRS-FW group failed by tendon rupture at the medial row rather than suture cutting through the tendon. Three samples in the DRS-FW group were failed by anchor pullout and one failed by the suture cutting the medial row anchor. Suture breakage turned out to be the most frequent failure mode in the DRS-Mesh group, with six out of eight specimens. Two samples in the DRS-Mesh group failed by tendon rupture at the medial row.

If we combine the modes of suture-cutting through tendon and tendon rupture as suture-tendon disconnection, the incidence rate of suture-tendon disconnection was $100 \%$ for the SB-FW group, $87.5 \%$ for SB-Mesh group, $50 \%$ for the DRS-FW group, and 25\% for the DRS-Mesh group. With the same suture used for the rotator cuff repair, the incidence rate of suture-tendon disconnection was $56.25 \%$ for mesh suture and $75.00 \%$ for the FiberWire. No significant difference was observed between the mesh suture and the FiberWire. With the same technique used for the rotator cuff repair, the incidence rate of the suture-tendon disconnection for SB technique $(93.75 \%)$ was significantly higher than that of DRS technique $(37.50 \%, \mathrm{P}<0.01)$.

\section{Maximum load to tensile failure}

The failure load was $168.0 \pm 72.4 \mathrm{~N}$ for the SB-Mesh group, $145.5 \pm 35.0 \mathrm{~N}$ for the SB-FW group, $259.4 \pm 60.5 \mathrm{~N}$ for the DRS-Mesh group, and $272.5 \pm 74.6 \mathrm{~N}$ for the DRSFW group (Figure 6B). No significant difference was found between the SB-Mesh and SB-FW groups nor the DRSMesh and DRS-FW groups (Figure 6B). The failure load was significantly increased when the rotator cuff was repaired with DRS compared with the SB technique ( $\mathrm{P}<0.05$ for all).

\section{Stiffness}

The stiffness was $11.1 \pm 5.2 \mathrm{~N} / \mathrm{mm}$ for the SB-Mesh group, 

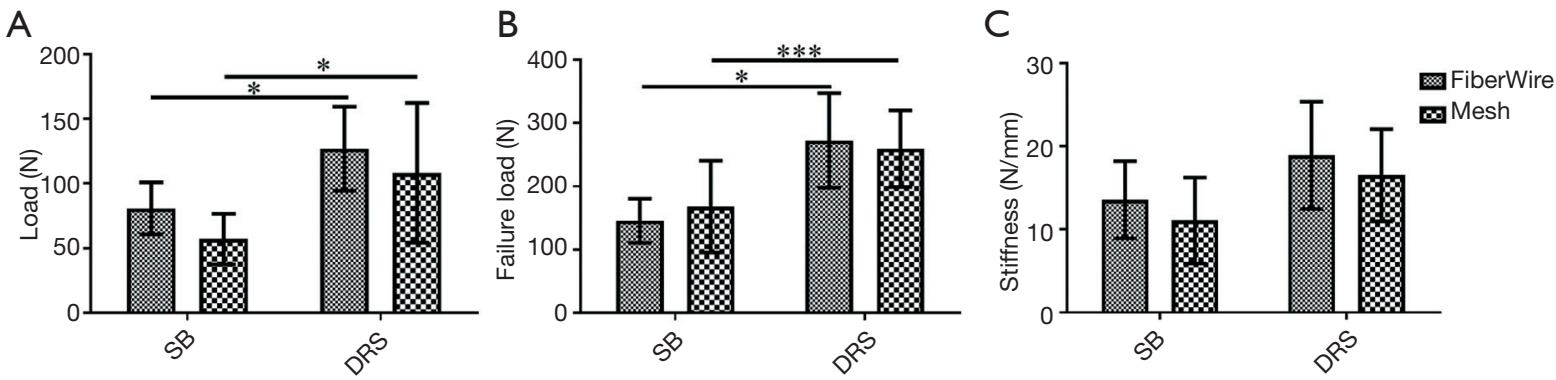

Figure 6 Comparison of the load to form a 3-mm gap (A), failure load (B), and stiffness (C) of the rotator cuff repaired with a mesh suture or a No. 2 FiberWire using a suture bridge and double rip-stop technique. SB, suture bridge; DRS, double rip-stop technique. * $\mathrm{P}<0.05$; ${ }^{* * *}$, $\mathrm{P}<0.001$.

Table 3 Failure modes of repaired rotator cuff

\begin{tabular}{|c|c|c|c|c|c|c|}
\hline Groups & $\begin{array}{l}\text { Suture cutting } \\
\text { through tendon }\end{array}$ & Tendon rupture & Anchor pullout & Suture breakage & Suture anchor disconnection & Total number \\
\hline SB-FW & 8 & - & - & - & - & 8 \\
\hline DRS-FW & - & 4 & 3 & - & 1 & 8 \\
\hline DRS-Mesh & - & 2 & - & 6 & - & 8 \\
\hline
\end{tabular}

$\mathrm{SB}$, suture bridge; DRS, double rip-stop technique; FW, FiberWire.

$13.6 \pm 4.6 \mathrm{~N} / \mathrm{mm}$ for the SB-FW group, $16.5 \pm 5.6 \mathrm{~N} / \mathrm{mm}$ for the DRS-Mesh group, and $18.9 \pm 6.5 \mathrm{~N} / \mathrm{mm}$ for the DRS-FW group (Figure 6C). No significant difference was observed between the SB-Mesh group and the SB-FW group nor the DRS-Mesh group and the DRS-FW group.

\section{Discussion}

In this current study, the results showed that the mesh suture had similar biomechanical effects on the suturetendon interface compared with No. 2 FiberWire with respect to failure load, stiffness, and ultimate stress. Furthermore, the repaired rotator cuff utilizing mesh suture demonstrated equivalent load to create a 3-mm gap, failure load, and stiffness compared with the No. 2 FiberWire under the condition that the same repair technique was applied. These are important findings, as repairs with a mesh suture have been found in in vivo animal models to improve over time with the ability of tissues to integrate around the filaments and into the body of the device (16). In comparison, as is well known, standard sutures tend to loosen immediately after placement due to softening of the tissues that they are holding.
Surgical techniques of rotator cuff repair have been developed over the last two decades. Many studies reported that the clinical outcome has been improved after surgery regarding pain relief and range of shoulder motion (14,33-36). The retear rate was reported regardless of the repair techniques (37-39). Therefore, efforts are still needed to improve the healing rate and to reduce the retear rate after rotator cuff repair surgery. The common failure modes observed in repaired rotator cuff include suture breakage, suture cutting through the tendon, sutureanchor disconnection, knot failure, and anchor pull-out from the bone (40). With significant advancement in the anchor and suture materials, the suture-tendon interface has been shown as the weakest point, resulting in the suture-tendon interface failure as the most common failure mode of the repaired rotator cuff $(31,41,42)$. Bisson et al. investigated the influence of different suture materials on biomechanical properties of suture-interface using a simple suture looptendon model (12). The results indicated that the suture material would influence early gap formation (12). However, in the present study, the type of suture materials did not influence the biomechanical properties of suture-tendon interface with the suture loop-tendon model. The possible 
reason was that the testing protocols used in the present and Bisson's studies were different. In Bisson's study, cyclic load testing was first performed then followed by failure load testing. In the present study, all the specimens were directly loaded to failure. Although there were significant differences among the groups regarding to the stiffness and peak-topeak displacement in Bisson's study, the differences were small in magnitude. Moreover, the failure load reported in Bisson's study was similar among the tested groups.

Mesh suture is specially fabricated with a hollow shape. Many studies have shown promising results with the application of mesh suture to hernia and abdomen surgeries (15-18,43-45). Afterwards, Zhang et al. assessed the biomechanical properties of three different suture materials including mesh suture, FiberWire, and polydioxanone suture II in a canine rotator cuff repair model (19). The results showed that the failure load of the rotator cuff repaired with Mesh suture was significantly higher than the other two groups. On the contrary, the current results demonstrated that there were no significant differences in the biomechanical properties between mesh suture and FiberWire under the same condition. One possible reason was that the testing protocol, where the repair constructs, was gradually loaded to failure by the tensiometer. The human cadaveric finger tendon study that showed improved outcomes using mesh suture over FiberWire employed a cyclic loading protocol (20). The other possible reason was that the diameters of mesh suture and FiberWire, as well as the techniques used in these two studies, were different. The diameter of the mesh suture and FiberWire used in Zhang's study was 1.7 and $0.35 \mathrm{~mm}$, respectively, which was 2.59 and $0.69 \mathrm{~mm}$ in this current study, respectively. Meanwhile, in Zhang's study, the simple interrupted and suture-bridge (SB) techniques were used. While in this present study, single-loop, SB, and DRS techniques were all assessed. The different suture diameters and repair techniques could contribute to the different results reported in these two studies. However, the failure load and load to create a 3-mm gap formation were significantly improved in the DRS technique compared with that of the SB technique when the same suture material was used. Furthermore, when the same technique was applied, the suture-tendon disconnection of SB technique was significantly higher than the DRS technique. This implied that the DRS technique enhanced the strength of the suture-tendon interface at the medial row and transferred the weak point of suture-tendon interface compared with the SB technique, thus increasing the initial repair strength.
The results of this current study were consistent with the previous study (25). Wu et al. reported that with a single rip-stop configuration, the failure load and stiffness were significantly improved compared with SB technique (25). However, the DRS technique takes more time than the commonly used SB technique. Whether it is worth the extra time to achieve the better biomechanical properties of the DRS technique needs further study. Weather the DRS could be performed arthroscopically also needs further investigation.

We acknowledged that there are some limitations for this current study. First, this current study tested the suture-tendon specimens and repaired rotator cuff at time zero. The rotator cuff healing after repair in vivo is a complicated biological process. Though the mesh suture has been shown to incorporate thereby creating a scar scaffold for healing (16), whether the mesh suture could improve the rotator cuff healing in vivo needs to be further studied. Second, the 0 sized mesh suture was chosen for the repair. It is possible that a larger sized mesh suture, or a mesh suture with thicker polypropylene filaments designed for orthopedic indications, would have improved outcomes in this ex vivo model. Third, the use of a porcine model limits the direct translation of our results to human rotator cuff repairs. Since the quality of human specimens is hard to control, the use of porcine rotator cuff had better consistency with easier availability. This would be helpful to reduce the unknown factors caused by tendon quality. Fourth, as mentioned above, the failure testing without cyclic testing was not tested. Though the cyclic testing was more likely to simulate the shoulder movement, the initial strength after repair still played an important role to reduce retear in the early motion. All the repaired rotator cuff specimens were tested under the same condition, so the influence of the suture materials on the specimens was comparable and convincible.

\section{Conclusions}

The repair failure strength and stiffness using the mesh suture were similar to the FiberWire suture regardless of the repair techniques. However, the repair strength in the DRS technique was significantly stronger than the SB technique when the same suture material was used.

\section{Acknowledgments}

Funding: This study received funding from NIH/NIAMS 
(AR073811-01A1).

\section{Footnote}

Reporting Checklist: The authors have completed the MDAR reporting checklist. Available at http://dx.doi.org/10.21037/ atm-20-1065

Data Sharing Statement: Available at http://dx.doi. org/10.21037/atm-20-1065

Conflicts of Interest: All authors have completed the ICMJE uniform disclosure form (available at http://dx.doi. org/10.21037/atm-20-1065). CZ reports grants from NIH/ NIAMS (AR073811-01A1), during the conduct of the study. The other authors have no conflicts of interest to declare.

Ethical Statement: All the samples were harvested from pigs sacrificed for other studies approved by the Institutional Animal Care and Use Committee of Mayo Clinic. The authors are accountable for all aspects of the work in ensuring that questions related to the accuracy or integrity of any part of the work are appropriately investigated and resolved.

Open Access Statement: This is an Open Access article distributed in accordance with the Creative Commons Attribution-NonCommercial-NoDerivs 4.0 International License (CC BY-NC-ND 4.0), which permits the noncommercial replication and distribution of the article with the strict proviso that no changes or edits are made and the original work is properly cited (including links to both the formal publication through the relevant DOI and the license). See: https://creativecommons.org/licenses/by-nc-nd/4.0/.

\section{References}

1. Yamaguchi K, Ditsios K, Middleton WD, et al. The demographic and morphological features of rotator cuff disease. A comparison of asymptomatic and symptomatic shoulders. J Bone Joint Surg Am 2006;88:1699-704.

2. Boileau P, Brassart N, Watkinson DJ, et al. Arthroscopic repair of full-thickness tears of the supraspinatus: Does the tendon really heal? J Bone Joint Surg Am 2005;87:1229-40.

3. Kwon DR, Park GY. Adult mesenchymal stem cells for the treatment in patients with rotator cuff disease: present and future direction. Ann Transl Med 2018;6:432.

4. Nho SJ, Brown BS, Lyman S, et al. Prospective analysis of arthroscopic rotator cuff repair: prognostic factors affecting clinical and ultrasound outcome. J Shoulder Elbow Surg 2009;18:13-20.

5. Oh JH, Chung SW, Oh KS, et al. Effect of recombinant human growth hormone on rotator cuff healing after arthroscopic repair: preliminary result of a multicenter, prospective, randomized, open-label blinded end point clinical exploratory trial. J Shoulder Elbow Surg 2018;27:777-85.

6. Kim YS, Sung CH, Chung SH, et al. Does an Injection of Adipose-Derived Mesenchymal Stem Cells Loaded in Fibrin Glue Influence Rotator Cuff Repair Outcomes? A Clinical and Magnetic Resonance Imaging Study. Am J Sports Med 2017;45:2010-8.

7. Fu CJ, Sun JB, Bi ZG, et al. Evaluation of platelet-rich plasma and fibrin matrix to assist in healing and repair of rotator cuff injuries: a systematic review and meta-analysis. Clin Rehabil 2017;31:158-72.

8. Saltzman BM, Jain A, Campbell KA, et al. Does the Use of Platelet-Rich Plasma at the Time of Surgery Improve Clinical Outcomes in Arthroscopic Rotator Cuff Repair When Compared With Control Cohorts? A Systematic Review of Meta-analyses. Arthroscopy 2016;32:906-18.

9. Ono Y, Davalos Herrera DA, Woodmass JM, et al. Can Grafts Provide Superior Tendon Healing and Clinical Outcomes After Rotator Cuff Repairs?: A Meta-analysis. Orthop J Sports Med 2016;4:2325967116674191.

10. Ferguson DP, Lewington MR, Smith TD, et al. Graft Utilization in the Augmentation of Large-to-Massive Rotator Cuff Repairs: A Systematic Review. Am J Sports Med 2016;44:2984-92.

11. Lorbach O, Baums MH, Kostuj T, et al. Advances in biology and mechanics of rotator cuff repair. Knee Surg Sports Traumatol Arthrosc 2015;23:530-41.

12. Bisson LJ, Manohar LM, Wilkins RD, et al. Influence of suture material on the biomechanical behavior of suturetendon specimens: a controlled study in bovine rotator cuff. Am J Sports Med 2008;36:907-12.

13. Bisson LJ, Manohar LM. A biomechanical comparison of the pullout strength of No. 2 FiberWire suture and 2-mm FiberWire tape in bovine rotator cuff tendons. Arthroscopy 2010;26:1463-8.

14. Noyes MP, Ladermann A, Denard PJ. Functional Outcome and Healing of Large and Massive Rotator Cuff Tears Repaired With a Load-Sharing Rip-Stop Construct. Arthroscopy 2017;33:1654-8.

15. Lanier ST, Dumanian GA, Jordan SW, et al. Mesh Sutured Repairs of Abdominal Wall Defects. Plast Reconstr Surg 
Glob Open 2016;4:e1060.

16. Souza JM, Dumanian ZP, Gurjala AN, et al. In vivo evaluation of a novel mesh suture design for abdominal wall closure. Plast Reconstr Surg 2015;135:322e-330e.

17. Agarwal BB, Agarwal KA, Sahu T, et al. Traditional polypropylene and lightweight meshes in totally extraperitoneal inguinal herniorrhaphy. Int J Surg 2010;8:44-7.

18. Guzmán-Valdivia G, Guerrero TS, Laurrabaquio HV. Parastomal hernia-repair using mesh and an open technique. World J Surg 2008;32:465-70.

19. Zhang T, Hatta T, Thoreson AR, et al. Rotator cuff repair with a novel mesh suture: An ex vivo assessment of mechanical properties. J Orthop Res 2018;36:987-92.

20. Wallace SJ, Mioton LM, Havey RM, et al. Biomechanical Properties of a Novel Mesh Suture in a Cadaveric Flexor Tendon Repair Model. J Hand Surg Am 2019;44:208-15.

21. Barber FA, Drew OR. A biomechanical comparison of tendon-bone interface motion and cyclic loading between single-row, triple-loaded cuff repairs and double-row, suture-tape cuff repairs using biocomposite anchors. Arthroscopy 2012;28:1197-205.

22. Awwad GE, Eng K, Bain GI, et al. Medial grasping sutures significantly improve load to failure of the rotator cuff suture bridge repair. J Shoulder Elbow Surg 2014;23:720-8.

23. Denard PJ, Burkhart SS. A load-sharing rip-stop fixation construct for arthroscopic rotator cuff repair. Arthrosc Tech 2012;1:e37-42.

24. Burkhart SS, Denard PJ, Konicek J, et al. Biomechanical validation of load-sharing rip-stop fixation for the repair of tissue-deficient rotator cuff tears. Am J Sports Med 2014;42:457-62.

25. Wu Z, Zhang C, Zhang P, et al. Biomechanical Comparison of Modified Suture Bridge Using Rip-Stop versus Traditional Suture Bridge for Rotator Cuff Repair. Biomed Res Int 2016;2016:9872643.

26. Najibi S, Banglmeier R, Matta J, et al. Material properties of common suture materials in orthopaedic surgery. Iowa Orthop J 2010;30:84-8.

27. Ma CB, MacGillivray JD, Clabeaux J, et al. Biomechanical evaluation of arthroscopic rotator cuff stitches. J Bone Joint Surg Am 2004;86:1211-6.

28. Lorbach O, Bachelier F, Vees J, et al. Cyclic loading of rotator cuff reconstructions: single-row repair with modified suture configurations versus double-row repair. Am J Sports Med 2008;36:1504-10.

29. Milano G, Grasso A, Zarelli D, et al. Comparison between single-row and double-row rotator cuff repair: a biomechanical study. Knee Surg Sports Traumatol Arthrosc 2008;16:75-80.

30. Pauly S, Kieser B, Schill A, et al. Biomechanical comparison of 4 double-row suture-bridging rotator cuff repair techniques using different medial-row configurations. Arthroscopy 2010;26:1281-8.

31. Pauly S, Fiebig D, Kieser B, et al. Biomechanical comparison of four double-row speed-bridging rotator cuff repair techniques with or without medial or lateral row enhancement. Knee Surg Sports Traumatol Arthrosc 2011;19:2090-7.

32. Burkhart SS. The deadman theory of suture anchors: observations along a south Texas fence line. Arthroscopy 1995;11:119-23.

33. Buckup J, Smolen D, Hess F, et al. The arthroscopic triple-row modified suture bridge technique for rotator cuff repair: functional outcome and repair integrity. $\mathrm{J}$ Shoulder Elbow Surg 2020;29:308-15.

34. Noyes MP, Ladermann A, Denard PJ. Functional Outcome and Healing With a Load-Sharing Rip-Stop Repair Compared With a Single-Row Repair for Large and Massive Rotator Cuff Tears. Arthroscopy 2019;35:2295-300.

35. Lee KW, Yang DS, Lee GS, et al. Clinical outcomes and repair integrity after arthroscopic full-thickness rotator cuff repair: suture-bridge versus double-row modified Mason-Allen technique. J Shoulder Elbow Surg 2018;27:1953-9.

36. Hantes ME, Ono Y, Raoulis VA, et al. Arthroscopic Single-Row Versus Double-Row Suture Bridge Technique for Rotator Cuff Tears in Patients Younger Than 55 Years: A Prospective Comparative Study. Am J Sports Med 2018;46:116-21.

37. Pulatkan A, Anwar W, Tokdemir S, et al. The clinical and radiologic outcome of microfracture on arthroscopic repair for full-thickness rotator cuff tear. J Shoulder Elbow Surg 2020;29:252-7.

38. Ma HH, Chen KH, Chiang ER, et al. Does Arthroscopic Suture-Spanning Augmentation of Single-Row Repair Reduce the Retear Rate of Massive Rotator Cuff Tear? Am J Sports Med 2019;47:1420-6.

39. Kwong CA, Ono Y, Carroll MJ, et al. Full-Thickness Rotator Cuff Tears: What Is the Rate of Tear Progression? A Systematic Review. Arthroscopy 2019;35:228-34.

40. Mazzocca AD, Millett PJ, Guanche CA, et al. Arthroscopic single-row versus double-row suture anchor rotator cuff repair. Am J Sports Med 2005;33:1861-8.

41. Hinse S, Menard J, Rouleau DM, et al. Biomechanical study comparing 3 fixation methods for rotator cuff 
massive tear: Transosseous No. 2 suture, transosseous braided tape, and double-row. J Orthop Sci 2016;21:732-8.

42. Mihata T, Fukuhara T, Jun BJ, et al. Effect of shoulder abduction angle on biomechanical properties of the repaired rotator cuff tendons with 3 types of double-row technique. Am J Sports Med 2011;39:551-6.

43. Dumanian GA, Lanier ST, Souza JM, et al. Mesh sutured repairs of contaminated incisional hernias. Am J Surg
2018;216:267-73.

44. Dumanian GA, Tulaimat A, Dumanian ZP. Experimental study of the characteristics of a novel mesh suture. Br J Surg 2015;102:1285-92.

45. Conze J, Kingsnorth AN, Flament JB, et al. Randomized clinical trial comparing lightweight composite mesh with polyester or polypropylene mesh for incisional hernia repair. Br J Surg 2005;92:1488-93.
Cite this article as: Wang Z, Long Z, Li H, Lu H, Gingery A, Amadio PC, Moran SL, Zhao C. A biomechanical comparison of a mesh suture to a polyblend suture in a porcine tendon model. Ann Transl Med 2021;9(6):450. doi: 10.21037/atm-201065 